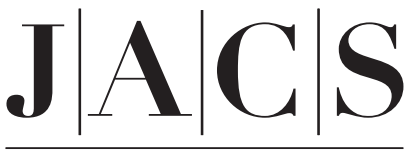

A R T I C L E S

Published on Web 11/19/2008

\title{
Large-Scale Synthesis of Single-Crystalline Iron Oxide Magnetic Nanorings
}

\author{
Chun-Jiang Jia, ${ }^{\dagger}$ Ling-Dong Sun, ${ }^{* \dagger}{ }^{\dagger}$ Feng Luo, ${ }^{*, \ddagger}$ Xiao-Dong Han, ${ }^{\S}$ \\ Laura J. Heyderman, ${ }^{\ddagger}$ Zheng-Guang Yan, ${ }^{\dagger}$ Chun-Hua Yan, ${ }^{,+}{ }^{+}$Kun Zheng, ${ }^{\S}$ \\ Ze Zhang, ${ }^{\S}$ Mikio Takano," Naoaki Hayashi," Matthias Eltschka, ${ }^{\perp}$ Mathias Kläui, ${ }^{\perp}$ \\ Ulrich Rüdiger, ${ }^{\perp}$ Takeshi Kasama, ${ }^{\otimes}$ Lionel Cervera-Gontard, ${ }^{\circ}$ \\ Rafal E. Dunin-Borkowski, ${ }^{\circ}$ George Tzvetkov,", and Jörg Raabe ${ }^{\#}$ \\ Beijing National Laboratory for Molecular Sciences, State Key Laboratory of Rare Earth \\ Materials Chemistry and Applications, PKU-HKU Joint Laboratory in Rare Earth Materials \\ and Bioinorganic Chemistry, Peking University, Beijing 100871, China, Laboratory for Micro- \\ and Nanotechnology, Paul Scherrer Institut, 5232 Villigen PSI, Switzerland, Institute of \\ Microstructure \& Properties of Advanced Materials, Beijing University of Technology, \\ Beijing 100022, China, Institute for Chemical Research, Kyoto University, Uji, \\ Kyoto-fu 611-0011, Japan, Fachbereich Physik, Universität Konstanz, Universitätsstrasse 10, \\ 78457 Konstanz, Germany, Department of Materials Science and Metallurgy, University of \\ Cambridge, Pembroke Street, Cambridge CB2 3QZ, United Kingdom, Center for Electron \\ Nanoscopy, Technical University of Denmark, DK-2800 Kongens Lyngby, Denmark, and Swiss \\ Light Source, Paul Scherrer Institut, 5232 Villigen PSI, Switzerland
}

Received July 4, 2008; E-mail: yan@pku.edu.cn; sun@pku.edu.cn; Feng.Luo@psi.ch

\begin{abstract}
We present an innovative approach to the production of single-crystal iron oxide nanorings employing a solution-based route. Single-crystal hematite $\left(\alpha-\mathrm{Fe}_{2} \mathrm{O}_{3}\right)$ nanorings were synthesized using a double anion-assisted hydrothermal method (involving phosphate and sulfate ions), which can be divided into two stages: (1) formation of capsule-shaped $\alpha-\mathrm{Fe}_{2} \mathrm{O}_{3}$ nanoparticles and (2) preferential dissolution along the long dimension of the elongated nanoparticles (the $c$ axis of $\alpha-\mathrm{Fe}_{2} \mathrm{O}_{3}$ ) to form nanorings. The shape of the nanorings is mainly regulated by the adsorption of phosphate ions on faces parallel to $c$ axis of $\alpha-\mathrm{Fe}_{2} \mathrm{O}_{3}$ during the nanocrystal growth, and the hollow structure is given by the preferential dissolution of the $\alpha-\mathrm{Fe}_{2} \mathrm{O}_{3}$ along the $c$ axis due to the strong coordination of the sulfate ions. By varying the ratios of phosphate and sulfate ions to ferric ions, we were able to control the size, morphology, and surface architecture to produce a variety of three-dimensional hollow nanostructures. These can then be converted to magnetite $\left(\mathrm{Fe}_{3} \mathrm{O}_{4}\right)$ and maghemite $\left(\gamma-\mathrm{Fe}_{2} \mathrm{O}_{3}\right)$ by a reduction or reduction-oxidation process while preserving the same morphology. The structures and magnetic properties of these single-crystal $\alpha-\mathrm{Fe}_{2} \mathrm{O}_{3}$, $\mathrm{Fe}_{3} \mathrm{O}_{4}$, and $\gamma-\mathrm{Fe}_{2} \mathrm{O}_{3}$ nanorings were characterized by various analytical techniques. Employing off-axis electron holography, we observed the classical single-vortex magnetic state in the thin magnetite nanorings, while the thicker rings displayed an intriguing three-dimensional magnetic configuration. This work provides an easily scaled-up method for preparing tailor-made iron oxide nanorings that could meet the demands of a variety of applications ranging from medicine to magnetoelectronics.
\end{abstract}

\section{Introduction}

In recent years, magnetic nanostructures have attracted significant attention, not only because of their fascinating physical properties but also because of their potential use in a range of applications, including magnetic random access memory, magnetic sensors, and logic devices. ${ }^{1-3}$ Ferromagnetic rings have particularly been the focus of interest because they have well-defined, reproducible magnetic states that result from

\footnotetext{
† Peking University.

¥ Laboratory for Micro- and Nanotechnology, Paul Scherrer Institut.

$\S$ Beijing University of Technology.

"Kyoto University.

${ }^{\perp}$ Universität Konstanz

${ }^{\otimes}$ University of Cambridge.

○ Technical University of Denmark.

\# Swiss Light Source, Paul Scherrer Institut.
}

their unique geometry. Such states are particularly interesting for industrial applications, since they can be easily detected and manipulated either in a magnetic field or with a spin-polarized current. $^{4}$

For applications involving current-induced changes of the magnetic states, nanorings made from a highly spin-polarized ferromagnetic material such as magnetite $\left(\mathrm{Fe}_{3} \mathrm{O}_{4}\right)$ would be a

(1) Wolf, S. A.; Awschalom, D. D.; Buhrman, R. A.; Daughton, J. M.; von Molnar, S.; Roukes, M. L.; Chtchelkanova, A. Y.; Treger, D. M. Science 2001, 294, 1488-1495.

(2) Cowburn, P.; Welland, M. E. Science 2000, 287, 1466-1468.

(3) Zhu, J. G.; Zheng, Y. F.; Prinz, G. A. J. Appl. Phys. 2000, 87, 66686673.

(4) Wen, Z. C.; Wei, H. X.; Han, X. F. Appl. Phys. Lett. 2007, 91, 122511$1-122511-3$. 
significant advantage. ${ }^{5}$ However, the preparation of singlecrystal magnetite rings using either physical or chemical fabrication routes has proven to be a challenge. Electron-beam lithography (EBL) has been widely used to fabricate magnetic nanorings from thin metallic films, ${ }^{6-8}$ but applying this structuring method to single-crystal films is difficult for two reasons: the polymer coating used as a mask during the lithographic processing deforms at the high temperatures required for singlecrystal film growth, and the different processing steps involved in EBL make it hard to produce high-quality films. With regard to chemical methods, it transpires that control of the synthesis of single-crystal $\mathrm{Fe}_{3} \mathrm{O}_{4}$ in solution is difficult because the magnetite is a metastable phase. ${ }^{9-11}$ Therefore, in order to provide a straightforward and robust process to produce $\mathrm{Fe}_{3} \mathrm{O}_{4}$ nanorings, we have developed a novel solution-based method involving a double anion-assisted hydrothermal route. This chemical route provides significantly larger volumes of material than EBL. In addition, while the thickness of the nanorings produced with EBL is limited, ${ }^{12,13}$ this solution-based method opens the possibility of creating a range of three-dimensional structures.

In this paper, we first present the controllable synthesis of single-crystal $\alpha-\mathrm{Fe}_{2} \mathrm{O}_{3}$ nanorings, which makes use of the cooperative action of phosphate and sulfate ions. The $\alpha-\mathrm{Fe}_{2} \mathrm{O}_{3}$ hollow nanostructures are then converted to magnetite $\left(\mathrm{Fe}_{3} \mathrm{O}_{4}\right)$ and maghemite $\left(\gamma-\mathrm{Fe}_{2} \mathrm{O}_{3}\right)$ by a subsequent reduction or reduction-oxidation process while preserving the nanoring morphology. The high-quality single-crystal nature of the $\mathrm{Fe}_{3} \mathrm{O}_{4}$ and $\gamma-\mathrm{Fe}_{2} \mathrm{O}_{3}$ structures is confirmed with various analytical techniques, and employing off-axis electron holography, we observe that the magnetic states in the magnetite rings are dependent on the ring thickness. The magnetic vortex state is present in the thinner rings, and there is a more complex threedimensional magnetic configuration in the thicker rings, indicating the transition from the vortex to the "tube" state. ${ }^{14}$

With the fabrication of iron oxide nanorings by double anion mediation, we provide a new strategy for large-scale fabrication of tailor-made magnetic nanostructures. The ability to create various three-dimensional morphologies with interesting magnetic properties enhances the prospects for application of magnetic nanostructures in a wide range of scientific and

(5) (a) Dedkov, Y. S.; Rüdiger, U.; Güntherodt, G. Phvs. Rev. B 2002, 65, 64417-1-64417-5. (b) Rothman, J.; Kläui, M.; Lopez-Diaz, L.; Vaz, C. A. F.; Bleloch, A.; Bland, J. A. C.; Cui, Z.; Speaks, R. Phvs. Rev. Lett. 2001, 86, 1098-1101.

(6) Li, S. P.; Peyrade, D.; Natali, M.; Lebib, A.; Chen, Y.; Ebels, U.; Buda, L. D.; Ounadjela, K. Phvs. Rev. Lett. 2001, 86, 1102-1105.

(7) Steiner, M.; Nitta, J. Appl. Phvs. Lett. 2004, 84, 939-941.

(8) Castano, F. J.; Ross, C. A.; Eilez, A.; Jung, W.; Frandsen, C. Phvs. Rev. B 2004, 69, 144421-1-144421-7.

(9) Caruntu, D.; Caruntu, G.; Chen, Y. X.; O'Connor, C. J.; Goloverda, G.; Kolesnichenko, V. L. Chem. Mater. 2004, 16, 5527-5534.

(10) He, Y. P.; Miao, Y. M.; Li, C. R.; Wang, S. Q.; Cao, L.; Xie, S. S.; Yang, G. Z.; Zou, B. S. Phvs. Rev. B 2005, 71, 125411-1-125411-9.

(11) Cha, H. G.; Lee, D. K.; Kim, Y. H.; Kim, C. W.; Lee, C. S.; Kang, Y. S. Inorg. Chem. 2008, 47, 121-127.

(12) Backes, D.; Heyderman, L. J.; David, C.; Schäublin, R.; Kläui, M.; Ehrke, H.; Rüdiger, U.; Vaz, C. A. F.; Bland, J. A. C.; Kasama, T.; Dunin-Borkowski, R. E. Microelectron. Eng. 2006, 83, 1726-1729.

(13) Heyderman, L. J.; David, C.; Kläui, M.; Vaz, C. A. F.; Bland, J. A. C. J. Appl. Phys. 2003, 93, 10011-10013.

(14) (a) Escrig, J.; Bachmann, J.; Jing, J.; Daub, M.; Altbir, D.; Nielsch, K. Phys. Rev. B 2008, 77, 214421-1-214421-7. (b) Escrig, J.; Landeros, P.; Altbir, D.; Vogel, E. E.; Vargas, P. J. Magn. Magn. Mater. 2007, 308, 233-237. technological fields, including medicine, biochemistry, and electronics. ${ }^{15-17}$

\section{Experimental Section}

Synthesis. $\alpha-\mathrm{Fe}_{2} \mathrm{O}_{3}$ Nanorings. The $\alpha-\mathrm{Fe}_{2} \mathrm{O}_{3}$ nanorings were prepared by a hydrothermal treatment of $\mathrm{FeCl}_{3}$ with additives. In a typical experimental procedure, specific amounts of $\mathrm{FeCl}_{3}$, $\mathrm{NaH}_{2} \mathrm{PO}_{4}$, and $\mathrm{Na}_{2} \mathrm{SO}_{4}$ aqueous solutions were mixed together, and then distilled water was added to the mixture to keep the final volume at $80 \mathrm{~mL}$; the concentrations of $\mathrm{FeCl}_{3}, \mathrm{NaH}_{2} \mathrm{PO}_{4}$, and $\mathrm{Na}_{2} \mathrm{SO}_{4}$ were $0.02,1.8 \times 10^{-4}$, and $5.5 \times 10^{-4} \mathrm{~mol} \mathrm{~L}^{-1}$, respectively. After vigorous stirring for $10 \mathrm{~min}$, the mixture was transferred into a Teflon-lined stainless steel autoclave with a capacity of $100 \mathrm{~mL}$ for hydrothermal treatment at $220{ }^{\circ} \mathrm{C}$ for $48 \mathrm{~h}$. After the autoclave was allowed to cool to room temperature, the precipitate was separated by centrifugation, washed with distilled water and absolute ethanol, and dried under vacuum at $80{ }^{\circ} \mathrm{C}$. In a single batch of experiments, more than $120 \mathrm{mg}$ of $\alpha-\mathrm{Fe}_{2} \mathrm{O}_{3}$ nanorings could be prepared. Varying the concentration of $\mathrm{NH}_{4} \mathrm{H}_{2} \mathrm{PO}_{4}$ and $\mathrm{Na}_{2} \mathrm{SO}_{4}$ produced a series of $\alpha-\mathrm{Fe}_{2} \mathrm{O}_{3}$ nanorings and nanotubes with different sizes and surface morphologies. In order to understand the roles of these two additives, we replaced the two additives of $\mathrm{NH}_{4} \mathrm{H}_{2} \mathrm{PO}_{4}$ and $\mathrm{Na}_{2} \mathrm{SO}_{4}$ by a single additive of either $\mathrm{NH}_{4} \mathrm{H}_{2} \mathrm{PO}_{4}$ or $\mathrm{Na}_{2} \mathrm{SO}_{4}$ while preserving all the other conditions described above for the preparation of the $\alpha-\mathrm{Fe}_{2} \mathrm{O}_{3}$ nanorings.

$\mathrm{Fe}_{3} \mathrm{O}_{4}$ and $\gamma-\mathrm{Fe}_{2} \mathrm{O}_{3}$ Nanorings. The $\mathrm{Fe}_{3} \mathrm{O}_{4}$ nanorings were prepared via a reduction process with the corresponding $\alpha-\mathrm{Fe}_{2} \mathrm{O}_{3}$ products as starting materials. The dried $\alpha-\mathrm{Fe}_{2} \mathrm{O}_{3}$ powders were annealed in a furnace at $360^{\circ} \mathrm{C}$ under a continuous hydrogen/argon gas flow $\left[\mathrm{H}_{2} /\left(\mathrm{H}_{2}+\mathrm{Ar}\right)=8 / 100\right]$ for $5 \mathrm{~h}$. Then the furnace was allowed to cool to room temperature while still under a continuous hydrogen gas flow. The $\gamma-\mathrm{Fe}_{2} \mathrm{O}_{3}$ nanostructures were obtained by oxidation of the $\mathrm{Fe}_{3} \mathrm{O}_{4}$ powders by exposure to air at $240{ }^{\circ} \mathrm{C}$ for $2 \mathrm{~h}$.

Characterization. The powder X-ray diffraction (XRD) patterns were recorded on a Rigaku D/MAX-2000 diffractometer using $\mathrm{Cu}$ $\mathrm{K} \alpha$ radiation $(\lambda=1.5418 \AA$ ). Scanning electron microscopy (SEM) images were taken using a DB-235 focused ion beam system. Transmission electron microscopy (TEM) images were taken on a JEOL 200CX transmission electron microscope under a working voltage of $160 \mathrm{kV}$. High-resolution TEM (HRTEM) images and selected-area electron diffraction (SAED) patterns were performed with a Philips Tecnai F30 FEG-TEM instrument operated at 300 $\mathrm{kV}$ and a JEOL 2010F FEG-TEM operated at $200 \mathrm{kV}$. The samples for TEM or SEM observations were created by dropping the ethanol suspension containing the uniformly dispersed iron oxide powders onto carbon-coated copper grids or silicon substrates, respectively. Mössbauer spectra were measured at $293 \mathrm{~K}$ using a conversional constant-acceleration spectrometer in transmission geometry with $30 \mathrm{mCi}{ }^{57} \mathrm{Co}$ in a Rh matrix as the source. The spectrometer was calibrated using a foil of iron, and isomer shift values are quoted relative to $\alpha$-Fe at $293 \mathrm{~K}$. The surface composition was determined by X-ray photoelectron spectroscopy (XPS) in an ion-pumped chamber (evacuated to $1.3 \times 10^{-8}$ Torr) using an Axis Ultra spectrometer equipped with a focused monochromatic X-ray source (Al Ko, $h v=1486.6 \mathrm{eV}$ ) at a power of $225 \mathrm{~W}$. A hemispherical analyzer collected the photoelectrons at an angle of $90^{\circ}$ to the surface. The binding energy (BE) for the samples was calibrated by setting the measured $\mathrm{BE}$ of $\mathrm{C} 1 \mathrm{~s}$ to $284.6 \mathrm{eV}$. High-resolution imaging combined with local X-ray absorption spectroscopy using scanning transmission X-ray microscopy (STXM) was conducted at the PolLux beamline at the Swiss Light Source (SLS) at the

(15) Gupta, A. K.; Rohan, R. N.; Vaidya, V. D.; Gupta, M. Nanomedicine 2007, 2, 23-39.

(16) Saiyeda, Z. M.; Bochiwala, C.; Gorasiaa, H.; Telanga, S. D.; Ramchand, C. N. Anal. Biochem. 2006, 356, 306-308.

(17) Chang, M. T.; Chou, L. J.; Hsieh, C. H.; Chueh, Y. L.; Wang, Z. L.; Murakami, Y.; Shindo, D. Adv. Mater. 2007, 19, 2290-2294. 
Scheme 1. Schematic Illustration of the Formation Process for Hematite Nanostructures Mediated by Phosphate and Sulfate lons ${ }^{a}$

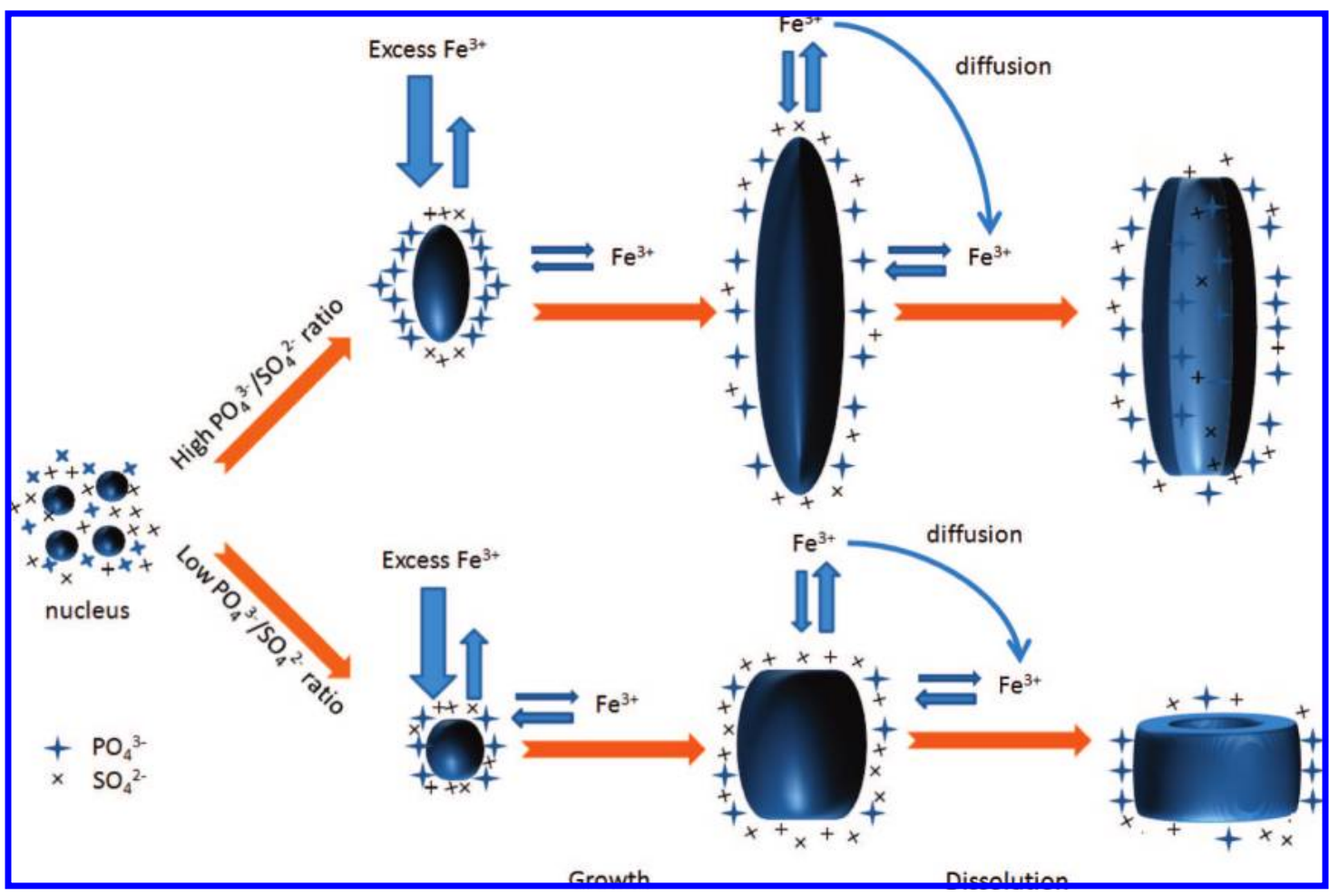

${ }^{a}$ Phosphate ions, with their strong affinity for (110) planes, act as a shape controller to induce anisotropic growth, while the sulfate ions, with their weak adsorption affinity, induce only the growth of polyhedra. With the two additives, the hematite precursors grow into capsule-shaped crystals. However, in the formation ematite nanorings, sulfate ions, with their relatively strong ability to coordinate to $\mathrm{Fe}^{3+}$ ions, promote the dissolution of the iron oxide capsules, and phosphate ions still act as a shape controller to keep the (001) anisotropy.

Paul Scherrer Institut. The PolLux STXM uses polarized X-rays from a bending magnet in the photon energy range between 200 and $1400 \mathrm{eV}$, providing a spatial resolution better than $40 \mathrm{~nm}$. The particles were deposited onto $\mathrm{Si}_{3} \mathrm{~N}_{4}$ membranes (Silson Ltd., 100 $\mathrm{nm}$ thickness) and imaged in transmission mode using a photomultiplier tube with a phosphor scintillator. Fe $L_{2,3}$-edge near-edge $\mathrm{X}$-ray absorption fine structure (NEXAFS) spectra were collected by performing image stack acquisition. Magnetic properties were investigated using a Quantum Design MPMS XL-5 SQUID magnetometer. The samples were placed in a low-susceptibility plastic sample holder for orientation-dependent measurements. The diamagnetic contribution from the sample holder was carefully determined and subsequently subtracted. Off-axis electron holography was used to study magnetic structures of the $\mathrm{Fe}_{3} \mathrm{O}_{4}$ nanorings at the nanometer scale. Measurements were performed at $300 \mathrm{kV}$ using a Philips CM300-ST FEG TEM equipped with a Lorentz lens, an electrostatic biprism, and a Gatan multiscan CCD camera. Holograms were acquired at remanence after saturation of the sample with in-plane fields of $\sim 10,000$ Oe. Details concerning the hologram-recording procedure used in this study and the processing used to extract phase information from the holograms are described elsewhere. ${ }^{18}$

\section{Results and Discussion}

1. $\alpha-\mathrm{Fe}_{2} \mathrm{O}_{3}$ Nanorings. Recently, we reported on the synthesis of single-crystalline $\alpha-\mathrm{Fe}_{2} \mathrm{O}_{3}$ nanotubes through a coordinationassisted dissolution process involving the selective adsorption

(18) (a) Kasama, T.; Moreno, M. S.; Dunin-Borkowski, R. E.; Newcomb, S. B.; Haberkorn, N.; Guimpel, J.; Midgley, P. A. Appl. Surf. Sci. 2006, 252, 3977-3983. (b) Dunin-Borkowski, R. E.; McCartney, M. R.; Smith, D. J. In Encyclopedia of Nanoscience and Nanotechnology; Nalwa, H. S., Ed.; American Scientific Publishers: Valencia, CA, 2004; Vol. 3, pp 41-100. of phosphate ions on hematite. ${ }^{19}$ However, the size and aspect ratio of the nanotubes prepared with a single phosphate additive could not be tuned effectively because the nanotubes only formed in a narrow range of the ratio of phosphate ion to ferric ions. Here we show that the introduction of sulfate ions is a good approach to better control the growth of hematite crystals. The $\alpha-\mathrm{Fe}_{2} \mathrm{O}_{3}$ nanorings were typically prepared through hydrothermal treatment of a solution of $\mathrm{FeCl}_{3}\left(0.020 \mathrm{~mol} \mathrm{~L}{ }^{-1}\right)$, $\mathrm{NH}_{4} \mathrm{H}_{2} \mathrm{PO}_{4}\left(1.8 \times 10^{-4} \mathrm{~mol} \mathrm{~L}{ }^{-1}\right)$, and $\mathrm{Na}_{2} \mathrm{SO}_{4}\left(5.5 \times 10^{-4}\right.$ $\mathrm{mol} \mathrm{L}{ }^{-1}$ ) at $220{ }^{\circ} \mathrm{C}$ for $48 \mathrm{~h}$. XRD patterns and Mössbauer spectra indicate that the $\alpha-\mathrm{Fe}_{2} \mathrm{O}_{3}$ rings are single-phase, and SEM and TEM images show their uniform shapes and sizes. HRTEM images confirm that the $\alpha-\mathrm{Fe}_{2} \mathrm{O}_{3}$ rings are singlecrystalline and have an axis orientation of $<001\rangle$. The proposed mechanism for the nanoring formation process is illustrated in Scheme 1. First, a capsule-shaped nanoparticle forms because of the strong adsorption of phosphate on faces parallel to the $c$ axis of $\alpha-\mathrm{Fe}_{2} \mathrm{O}_{3}$, and this determines the shape and size of the rings. Second, the dissolution of the nanoparticle along the $c$ axis of $\alpha-\mathrm{Fe}_{2} \mathrm{O}_{3}$ is favored by the cooperative coordination of sulfate and phosphate ions with ferric ions. Varying the ratios of phosphate and sulfate ions to ferric ions yields a series of hollow nanostructures with controlled size, morphology, and surface architecture.

1.1. Microstructural Characterization. In order to identify the structure phase and microstructure of the synthesized $\alpha-\mathrm{Fe}_{2} \mathrm{O}_{3}$ nanorings, we used several different techniques to characterize our products. $\alpha-\mathrm{Fe}_{2} \mathrm{O}_{3}$ consisting of nanorings with outer diameters of

(19) Jia, C. J.; Sun, L. D.; Yan, Z. G.; You, L. P.; Luo, F.; Han, X. D.; Pang, Y. C.; Zhang, Z.; Yan, C. H. Angew. Chem. Int. Ed 2005, 44, 4328-4333. 


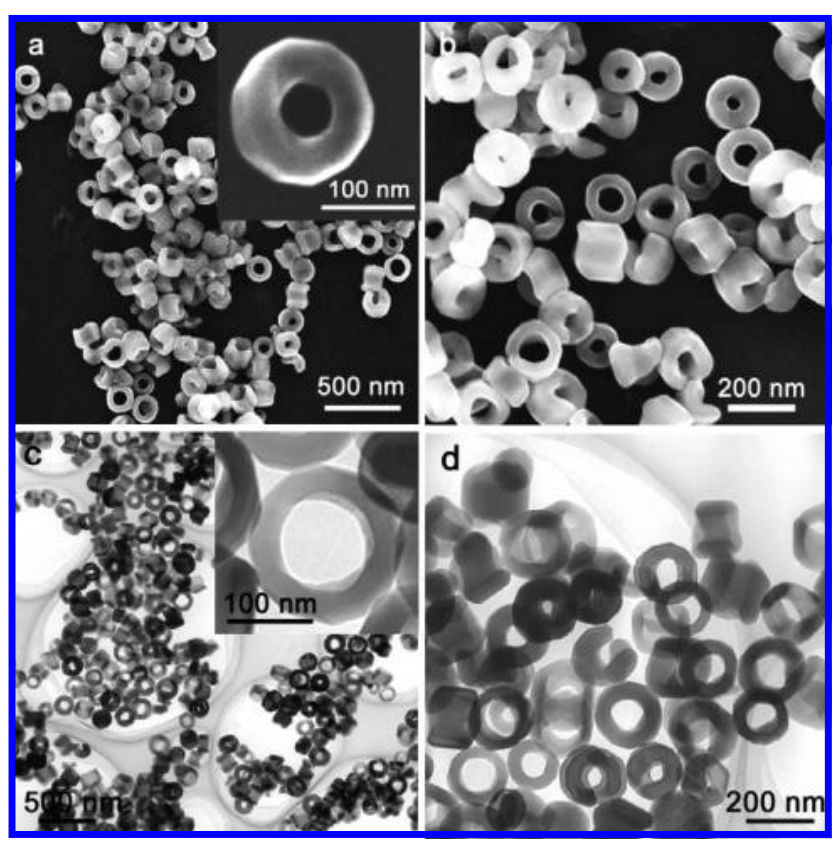

Figure 1. (a, b) SEM and (c, d) TEM images of the $\alpha-\mathrm{Fe}_{2} \mathrm{O}_{3}$ nanorings The nanorings are homogeneous and display a dodecagonal prismatic morphology.

$150-170 \mathrm{~nm}$, inner diameters of 70-100 nm, and heights of 80-120 $\mathrm{nm}$ are shown in SEM and TEM images at different magnifications (Figure 1). Low-magnification SEM images (Figure $\mathrm{S} 1$ in the Supporting Information) indicate that these nanorings are produced on a large scale with uniform size and morphology. The nanorings exhibit a dodecagonal prismatic morphology that differs from the common circular ${ }^{6}$ or hexagonal ${ }^{20}$ morphology, as can be discerned in the SEM and TEM images at higher magnification (insets of Figure 1a,c). XRD patterns of the products (Figure S2a in the Supporting Information) indicate that all of the peaks can be well-indexed to a pure corundum structure of hematite (JCPDS no. 33-0664) without any impurities. This single phase is further determined by the room-temperature Mössbauer spectrum (Figure S2b in the Supporting Information), which shows a single sextet with an isomeric shift $(\delta)$ of $0.374 \mathrm{~mm} \mathrm{~s}^{-1}$ (compared with that of standard $\alpha-\mathrm{Fe}$ ), a quadrupole splitting $(\varepsilon)$ of $-0.218 \mathrm{~mm}$ $\mathrm{s}^{-1}$, and a hyperfine field $\left(H_{\mathrm{hf}}\right)$ of $511.4 \mathrm{KOe}$ and thus provides clear evidence for the presence of $\alpha-\mathrm{Fe}_{2} \mathrm{O}_{3}$ rather than $\gamma-\mathrm{Fe}_{2} \mathrm{O}_{3}$ or $\mathrm{Fe}_{3} \mathrm{O}_{4}$.

From the top view of a single nanoring (Figure 2a), it can be seen that the product is a dodecagonal prismatic ring having a similarly shaped central hole. The SAED pattern (Figure 2b) indicates that the nanoring is a single crystal with a ring axis of [001]. On the basis of the SAED pattern, the external wall surfaces should be high-index planes, not the simple prismatic $\{110\}$ or $\{100\}$ planes of hexagonal hematite. The HRTEM images of the external wall (Figure $2 \mathrm{~d}$ ) show that there are many steps on the edges of the wall surfaces that can be indexed to (110) and $(\overline{2} 10)$ planes. Therefore, none of exposed external wall surface of the ring is a high-index plane; instead, the surface is a combination of low-energy $(\overline{110})$ and $(\overline{2} 10)$ planes belonging to $\{100\}$ and $\{110\}$, respectively, as marked in Figure $2 d$. The central hole of the nanorings has an irregular shape, and the HRTEM image (Figure 2c) indicates that the inner wall surfaces are composed of (110), $(\overline{2} 10)$, and (100) planes; the former two

(20) Li, F.; Ding, Y.; Gao, P. X.; Xin, X. Q.; Wang, Z. L. Angew. Chem. Int. Ed. 2004, 43, 5238-5242.

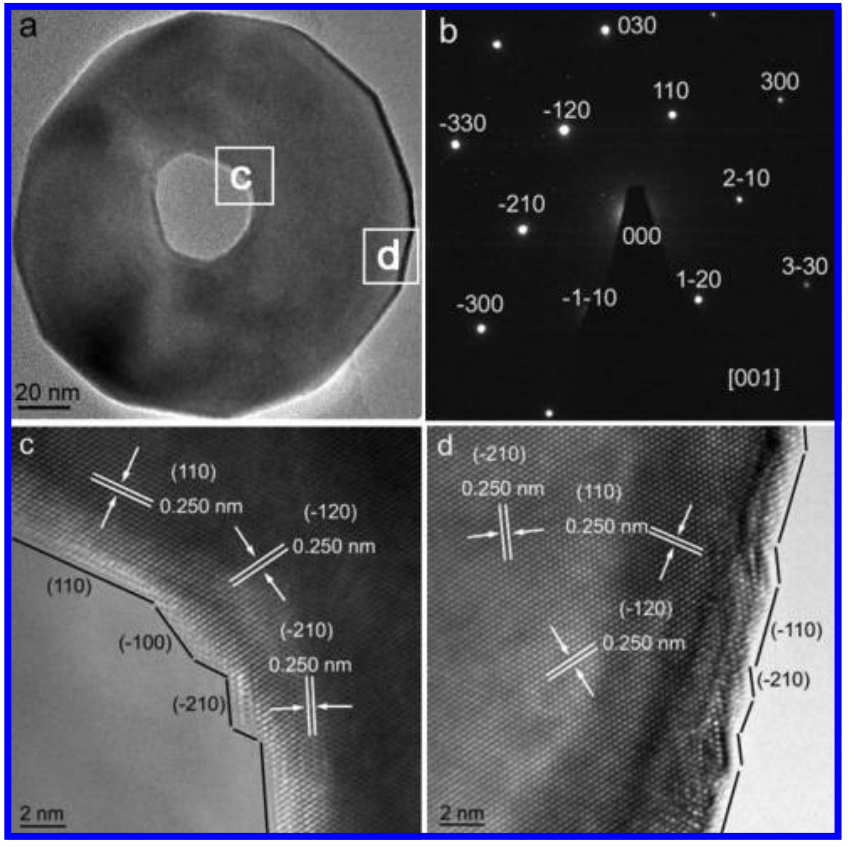

Figure 2. (a) TEM image, (b) ED pattern, and (c, d) HRTEM images of an $\alpha-\mathrm{Fe}_{2} \mathrm{O}_{3}$ nanoring indicate that the single-crystal nanoring grows along [001].

belong to $\{110\}$, and the latter belongs to $\{100\}$, similar to the outer wall surfaces. In order to lower the surface energy, the stable low-index facets of $\{110\}$ and $\{100\}$ are introduced instead of the unstable high-index planes, which give the formation of the dodecagonal prismatic nanorings.

1.2. Formation Mechanism of the $\alpha-\mathrm{Fe}_{2} \mathrm{O}_{3}$ Nanorings. The formation mechanism of the nanorings follows a preferential dissolution process, similar to that of hematite nanotubes (Figure 3). ${ }^{19}$ From the SEM images of the products taken as the reaction time increased (Figure $3 \mathrm{a}-\mathrm{e}$ ), it can be seen that the $\alpha-\mathrm{Fe}_{2} \mathrm{O}_{3}$ nanorings undergo an evolution from capsule-shaped nanoparticles to short nanorods and then to nanorings. The SAED pattern of the capsule-shaped nanoparticles at a reaction time of $2 \mathrm{~h}$ (Figure S3 in the Supporting Information) confirms that the nanoparticles grow along the [001] direction, which is the same as the axis of the nanorings in Figure 1, indicating that the growth direction does not change during the evolution process. It is noteworthy that the diameter of the final nanorings is larger and the surface is smoother than those of the capsule-shaped precursors, which indicates that recrystallization on the surface also accompanies the "dissolution" process. For reaction times extended to longer than $168 \mathrm{~h}$, the central holes of the nanorings become smaller and finally disappear, with the nanocrystals evolving into nanoprisms (Figure 3f). From the viewpoint of thermodynamics, it is the crystal structure rather than the additive (phosphate or sulfate ions) that determines the final morphology. $\alpha-\mathrm{Fe}_{2} \mathrm{O}_{3}$ has a rhombohedrally centered hexagonal structure of corundum, and the most common growth habits for $\alpha-\mathrm{Fe}_{2} \mathrm{O}_{3}$ are rhombohedral, platy, and rounded crystals. ${ }^{21}$ The nanoprisms shown in Figure $3 \mathrm{f}$ can be regarded as disks or plates because of their low aspect ratio, which is very consistent with the platy habit of $\alpha-\mathrm{Fe}_{2} \mathrm{O}_{3}$.

It is known that phosphate and sulfate ions can adsorb on the surface of $\alpha-\mathrm{Fe}_{2} \mathrm{O}_{3}(\{110\}$ and $\{100\})$, mainly by forming

(21) Cornell, R. M.; Schwertmann, U. The Iron Oxides: Structure, Properties, Reactions, Occurrence and Uses; VCH: Weinheim, Germany, 1996; p 74. 


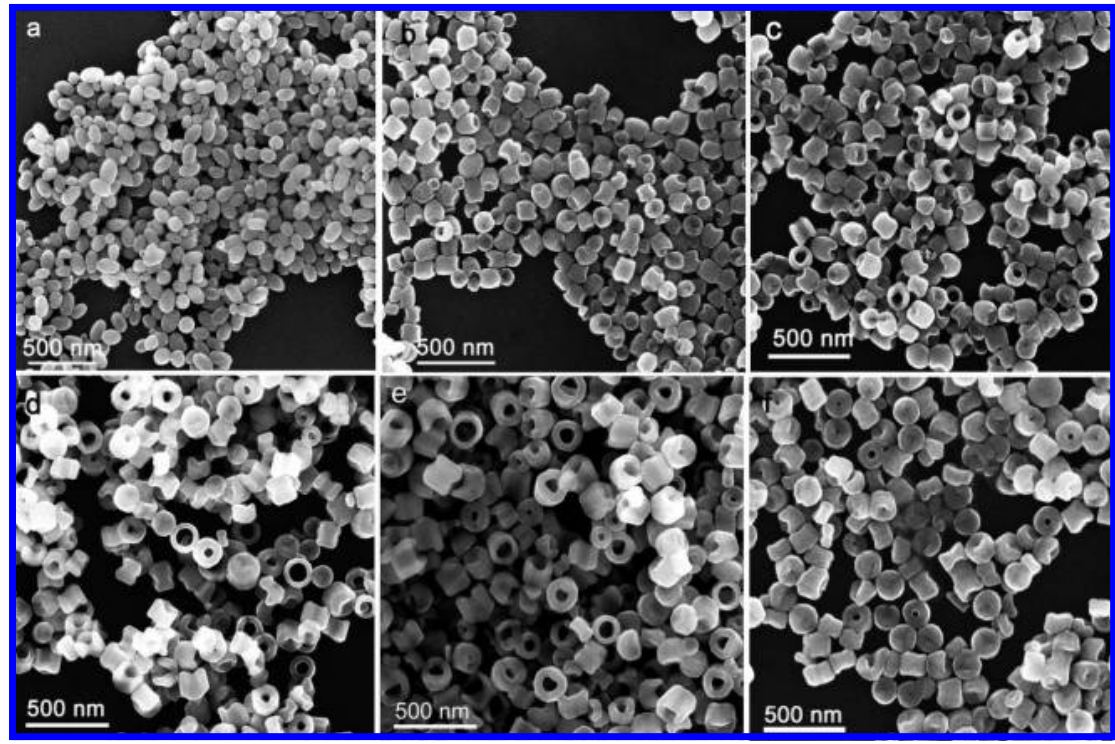

Figure 3. Morphological evolution of the $\alpha-\mathrm{Fe}_{2} \mathrm{O}_{3}$ nanorings with reaction time, as revealed by SEM images of the products prepared at $220{ }^{\circ} \mathrm{C}$ for (a) 2 , (b) 3 , (c) 6 , (d) 24 , (e) 48, and (f) $168 \mathrm{~h}$

binuclear, bidentate complexes with two singly coordinated hydroxyl groups, although other kinds of surface complexes may also be expected. ${ }^{22}$ The binuclear, bidentate adsorption of phosphate and sulfate ions on $\alpha-\mathrm{Fe}_{2} \mathrm{O}_{3}$ is firmly anchored, which could effectively prevent the detachment of iron atoms from the surfaces. On the basis of the schematic crystal structure of $\alpha-\mathrm{Fe}_{2} \mathrm{O}_{3}$ projected on different lattice planes (Figure $\mathrm{S} 4$ in the Supporting Information), pairs of singly coordinated hydroxyl groups are located on the prism planes of (110) and (100). However, the adsorption is very weak for the (001) plane because of the lack of singly coordinated hydroxyl groups. ${ }^{22}$ Thus, in the presence of phosphate and sulfate ions, the growth of the prism planes, (110) and (100), is restrained, and the $\alpha-\mathrm{Fe}_{2} \mathrm{O}_{3}$ nanoparticles grow along [001] direction, which induces the appearance of capsule-shaped structure. This is consistent with the previous results on the formation of spindlelike $\alpha-\mathrm{Fe}_{2} \mathrm{O}_{3}$ particles growing along [001] by the adsorption of phosphate ions. ${ }^{23}$ Under acidic conditions $(\mathrm{pH}$ $\sim 1.8$ ) at a high reaction temperature $\left(220^{\circ} \mathrm{C}\right)$, the tips of capsule-shaped nanoparticles may begin to dissolve in the following manner:

$$
\mathrm{Fe}_{2} \mathrm{O}_{3}+6 \mathrm{H}^{+} \rightarrow 2 \mathrm{Fe}^{3+}+3 \mathrm{H}_{2} \mathrm{O}
$$

The dissolution occurs along the [001] direction, as the (001) plane is almost entirely exposed to solution. HRTEM analysis of the nanorings has shown that the inner and external wall surfaces of the rings are composed of $\{110\}$ and $\{100\}$ planes (Figure 2), so the wall surfaces are effectively protected by the adsorption of phosphate and sulfate ions. Therefore, the formation of rings occurs through a process of preferential dissolution along the $c$ axis. Moreover, phosphate and sulfate ions can accelerate the dissolution by their coordination effect with detached ferric ions as follows:

$$
\begin{gathered}
\mathrm{Fe}^{3+}+x \mathrm{H}_{2} \mathrm{PO}_{4}{ }^{-} \rightarrow\left[\mathrm{Fe}\left(\mathrm{PO}_{4} \mathrm{H}_{2}\right)_{x}\right]^{3-x} \\
\mathrm{Fe}^{3+}+x \mathrm{SO}_{4}^{2-} \rightarrow\left[\mathrm{Fe}\left(\mathrm{SO}_{4}\right)_{x}\right]^{3-2 x}
\end{gathered}
$$

Therefore, in our work, the $\alpha-\mathrm{Fe}_{2} \mathrm{O}_{3}$ nanorings are formed by the cooperative action (adsorption and coordination) of the phosphate and sulfate ions.
Although the selectivities for adsorption of the phosphate and sulfate ions to $\alpha-\mathrm{Fe}_{2} \mathrm{O}_{3}$ crystal planes is similar, ${ }^{23}$ the adsorption affinity of sulfate on $\alpha-\mathrm{Fe}_{2} \mathrm{O}_{3}$ is much weaker than that of phosphate. $^{24}$ Therefore, the roles that phosphate and sulfate ions play in the formation of the nanorings should be different. In order to elucidate the roles of these two additives, the reactions using only phosphate or sulfate ions were investigated separately. Unlike the nanorings, either short nanorods or nanopolyhedra were formed with only phosphate ions $\left(1.8 \times 10^{-4} \mathrm{~mol}\right.$ $\left.\mathrm{L}^{-1}\right)$ or only sulfate ions $\left(5.5 \times 10^{-4} \mathrm{~mol} \mathrm{~L}^{-1}\right)$ as the additive, respectively (Figure S5a,b in the Supporting Information). TEM and ED investigations (Figure S5c,d in the Supporting Information) indicate that the anisotropic growth direction of the short nanorods is [001], which is mainly attributed to the selective adsorption of phosphate ions on surfaces parallel to the $c$ axis. By increasing the ratio of phosphate ions to ferric ions, nanoparticles, nanorods, and a mixture of nanorods and nanotubes were obtained, as shown in Figure S6 in the Supporting Information. The aspect ratios of these products increased with the ratio of phosphate ions to ferric ions, while only a few hollow nanostructures were formed, indicating that most of the phosphate ions act as a shape controller to induce the anisotropic growth, as shown in Scheme 1. Comparing Figure S5a in the Supporting Information to Figure $3 a$, we can see that the short nanorods and the capsule-shaped nanoparticles are quite similar in shape, aspect ratio, and growth direction. In comparison with phosphate, the adsorption affinity of sulfate to $\alpha-\mathrm{Fe}_{2} \mathrm{O}_{3}$ is much weaker. The SEM image of the $\alpha-\mathrm{Fe}_{2} \mathrm{O}_{3}$ products prepared only with sulfate ions $\left(5.5 \times 10^{-4} \mathrm{~mol} \mathrm{~L}^{-1}\right)$ is shown in Figure S5b in the Supporting Information. The products were composed of nanopolyhedra without obvious anisotropic growth. Adjusting the ratio of sulfate ions to ferric ions does not cause a change

(22) (a) Cornell, R. M.; Schwertmann, U. The Iron Oxides: Structure, Properties, Reactions, Occurrence and Uses; VCH: Weinheim, Germany, 1996; p 247. (b) Barrón, V.; Torrent, J. J. Colloid Interface Sci. 1996, 177, 407-410. (c) Huang, X. J. Colloid Interface Sci. 2004, 271, 296-307.

(23) Reeves, N. J.; Mann, S. J. Chem. Soc.. Faradav Trans. 1991, 87, 38753879.

(24) Sugimoto, T.; Wang, Y. S. J. Colloid Interface Sci. 1998, 207, $137-$ 149 . 
in particle shape, and there is no preferential anisotropic growth (Figure S7 in the Supporting Information), which indicates that the sulfate ions with their weak adsorption affinity cannot modify the crystal growth (Scheme 1). Therefore, because of their stronger adsorption effect, phosphate ions play a much more crucial role than sulfate ions in the formation of capsule-shaped nanoparticles in the first stage of the $\alpha-\mathrm{Fe}_{2} \mathrm{O}_{3}$ nanoring formation process. As a ligand, sulfate ions favor the dissolution of $\alpha-\mathrm{Fe}_{2} \mathrm{O}_{3}$ because of their coordination effect with ferric ions (eq 3), resulting in the final formation of a hollow structure.

The respective role of phosphate and sulfate ions shown in Scheme 1 was primarily deduced on the basis of the above analysis. The formation of the capsule-shaped nanoparticles, which determines the shape and size of the rings, is mainly controlled by the strong adsorption of phosphate on faces parallel to the $c$ axis of hematite, while the dissolution of the precursors is favored by the cooperative coordination effect of sulfate and phosphate ions with ferric ions, as shown in eqs 2 and 3 . Because of the strong adsorption, it is likely that phosphate mostly acts as an adsorbent on the surfaces of the hematite crystals, leading to a lower concentration of dissociated phosphate ions than dissociated sulfate ions. Therefore, in the dissolution process, sulfate ions play a more important role than phosphate ions. It is the effective cooperation between phosphate and sulfate ions that induces and favors the formation of the $\alpha-\mathrm{Fe}_{2} \mathrm{O}_{3}$ nanorings. It is noted that for longer reaction times $(\geq 168 \mathrm{~h})$, adsorption and desorption of phosphate or sulfate ions on the surface of $\alpha-\mathrm{Fe}_{2} \mathrm{O}_{3}$ reach an equilibrium, and the dissolution and recrystallization processes also gradually reach an equilibrium. $\alpha-\mathrm{Fe}_{2} \mathrm{O}_{3}$ tends to exhibit the thermodynamically stable shape of prism-like (disklike) crystals.

Experimental parameters such as temperature and $\mathrm{pH}$ are crucial for the formation of the nanorings. We found that lower temperatures $\left(<220^{\circ} \mathrm{C}\right)$ do not favor the formation of uniform nanorings because of the lack of sufficient energy, but higher temperatures favor overgrowth of the nanorings, resulting in narrow central holes and shorter lengths (Figure S8a,b in the Supporting Information). Moreover, higher $\mathrm{pH}$ values $(>2.5)$ $\alpha-\mathrm{Fe}_{2} \mathrm{O}_{3}$ are also not recommended because the dissolution process is prohibited as a result of the low concentration of protons (Figure S8c in the Supporting Information).

1.3. Size and Surface Control of $\alpha-\mathrm{Fe}_{2} \mathrm{O}_{3}$ Hollow Nanostructures. On the basis of the formation mechanism of the $\alpha-\mathrm{Fe}_{2} \mathrm{O}_{3}$ nanorings discussed above, it is reasonable to postulate that we can control the formation of the capsule-shaped nanoparticles and the subsequent dissolution process by varying the phosphate/sulfate ratio to tune the size and surface architecture of the $\alpha-\mathrm{Fe}_{2} \mathrm{O}_{3}$ nanostructures. Experiments were conducted with a fixed concentration of sulfate ions but various amounts of phosphate, and a series of $\alpha-\mathrm{Fe}_{2} \mathrm{O}_{3}$ nanostructures including nanorings, short nanotubes, and long nanotubes were prepared, as shown in Figure 4. The HRTEM images (Figure S9 in the Supporting Information) confirm the single-crystal nature of these hollow nanostructures. Similar to the nanorings in Figure 1, they all grow along the [001] direction. However, in contrast to the nanorings in Figure 1, those in Figure 4a are shorter along the ring axis, implying that the anisotropic growth along [001] is weakened as a result of the lower concentration of phosphate ions. With an increase in the amount of phosphate ions, the aspect ratio of the products increases, and nanotubes are eventually formed (Figure $4 \mathrm{~b}-\mathrm{d}$ ), further confirming that the phosphate ions are responsible for the growth of the capsule-

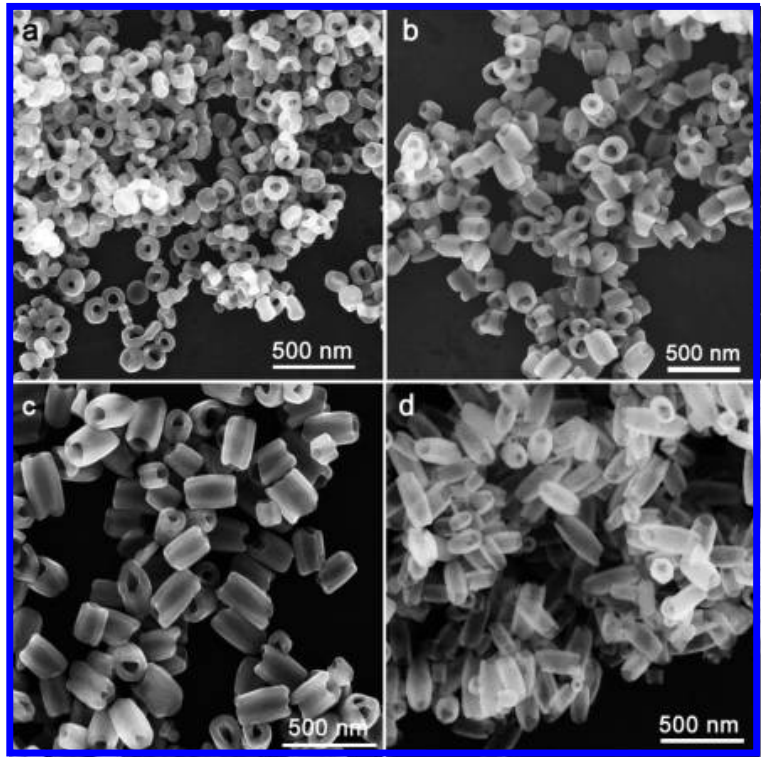

Figure 4. $\mathrm{SEM}$ images of $\alpha-\mathrm{Fe}_{2} \mathrm{O}_{3}$ products prepared with different concentrations of phosphate: (a) $\left[\mathrm{NH}_{4} \mathrm{H}_{2} \mathrm{PO}_{4}\right]=1.0 \times 10^{-4} \mathrm{~mol} \mathrm{~L}^{-1}$; (b) $\left[\mathrm{NH}_{4} \mathrm{H}_{2} \mathrm{PO}_{4}\right]=3.6 \times 10^{-4} \mathrm{~mol} \mathrm{~L}^{-1}$; (c) $\left[\mathrm{NH}_{4} \mathrm{H}_{2} \mathrm{PO}_{4}\right]=5.0 \times 10^{-4} \mathrm{~mol}$ $\mathrm{L}^{-1}$; (d) $\left[\mathrm{NH}_{4} \mathrm{H}_{2} \mathrm{PO}_{4}\right]=6.0 \times 10^{-4} \mathrm{~mol} \mathrm{~L}^{-1}$. In each of the preparations, $\left[\mathrm{FeCl}_{3}\right]=0.02 \mathrm{~mol} \mathrm{~L}^{-1}$ and $\left[\mathrm{Na}_{2} \mathrm{SO}_{4}\right]=5.5 \times 10^{-4} \mathrm{~mol} \mathrm{~L}^{-1}$.

shaped nanoparticles, which consequently determines the sizes and aspect ratios of the final hollow nanostructures.

Besides controlling the size and aspect ratio of the hollow nanostructures, varying the concentration of the sulfate ions could also modulate the surface architectures. SEM images of the nanotubes formed using a fixed concentration of phosphate ions and different amounts of sulfate ions (Figure S10 in the Supporting Information) indicate that with increasing sulfate concentration, the surfaces of the nanotubes became coarser (Figure S10b in the Supporting Information) and eventually became porous (Figure S10c,d in the Supporting Information). Although the wall surfaces of the nanotubes were coarse and even destroyed, the nanotubes were still single-crystalline in nature, as proved in Figure S10e, fin the Supporting Information.

2. Magnetic Nanorings $\left(\mathrm{Fe}_{3} \mathrm{O}_{4}\right.$ and $\left.\boldsymbol{\gamma}-\mathrm{Fe}_{2} \mathrm{O}_{3}\right)$. Here, $\alpha-\mathrm{Fe}_{2} \mathrm{O}_{3}$ nanorings were converted to $\mathrm{Fe}_{3} \mathrm{O}_{4}$ at $360{ }^{\circ} \mathrm{C}$ under a continuous hydrogen gas flow for $5 \mathrm{~h}$. The black powders obtained are $\mathrm{Fe}_{3} \mathrm{O}_{4}$ products. The $\gamma-\mathrm{Fe}_{2} \mathrm{O}_{3}$ nanostructures are obtained by oxidation of the as-prepared $\mathrm{Fe}_{3} \mathrm{O}_{4}$ powders via exposure to air at $240{ }^{\circ} \mathrm{C}$ for $2 \mathrm{~h}$. The morphology of the $\alpha-\mathrm{Fe}_{2} \mathrm{O}_{3}$ nanorings is perfectly preserved on conversion to $\mathrm{Fe}_{3} \mathrm{O}_{4}$ and $\gamma-\mathrm{Fe}_{2} \mathrm{O}_{3}$. The conversion is highly efficient, and a large quantity of $\mathrm{Fe}_{3} \mathrm{O}_{4}$ nanorings can be produced. From the XRD patterns combined with XPS, we identified the single-crystal structures of $\mathrm{Fe}_{3} \mathrm{O}_{4}$ and $\gamma-\mathrm{Fe}_{2} \mathrm{O}_{3}$. All of the $\mathrm{Fe}_{3} \mathrm{O}_{4}$ nanorings have the same chemical structure, as confirmed by the $\mathrm{Fe} L$-edge absorption spectra taken from several rings. HRTEM indicates two ring-axis orientations of $<111>$ and $<112>$ for the $\mathrm{Fe}_{3} \mathrm{O}_{4}$ nanorings, both of which show similar magnetic flux closure vortex states as observed with electron holography. These results show that it is the ring geometry rather than the magnetocrystalline anisotropy that determines the magnetic configuration of the single-crystal $\mathrm{Fe}_{3} \mathrm{O}_{4}$ nanorings. ${ }^{25}$ In the thicker nanorings, we observed a complicated

(25) Eltschka, M.; Kläui, M.; Rüdiger, U.; Kasama, T.; Cervera-Gontard, L.; Dunin-Borkowski, R. E.; Luo, F.; Heyderman, L. J.; Jia, C.-J.; Sun L.-D.; Yan, C.-H. Appl. Phys. Lett. 2008, 92, 222508-1-222508-3. 


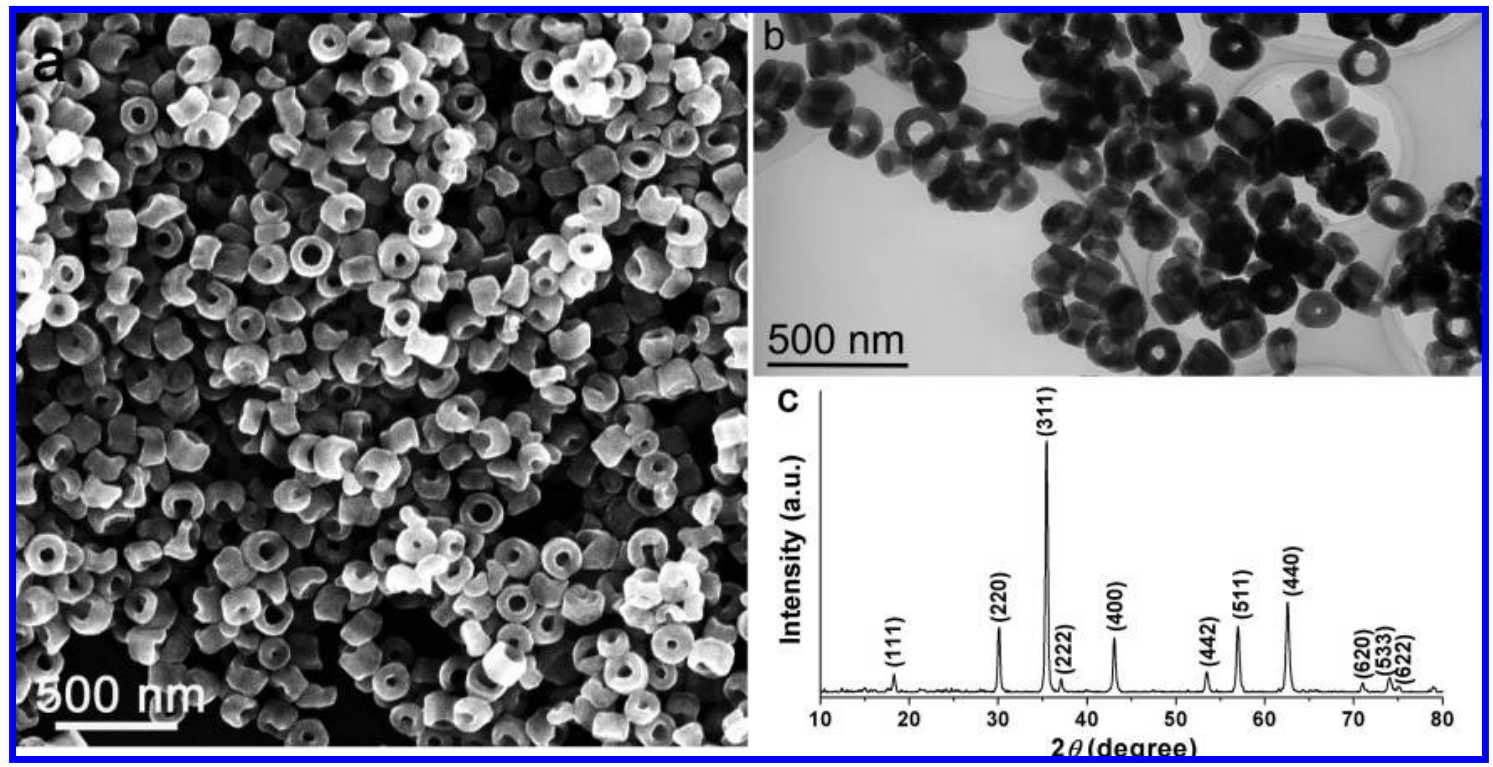

Figure 5. (a) SEM images, (b) TEM images, and (c) XRD patterns of the $\mathrm{Fe}_{3} \mathrm{O}_{4}$ nanorings. The morphology of the initial nanorings was maintained during the conversion from $\alpha-\mathrm{Fe}_{2} \mathrm{O}_{3}$ to $\mathrm{Fe}_{3} \mathrm{O}_{4}$.

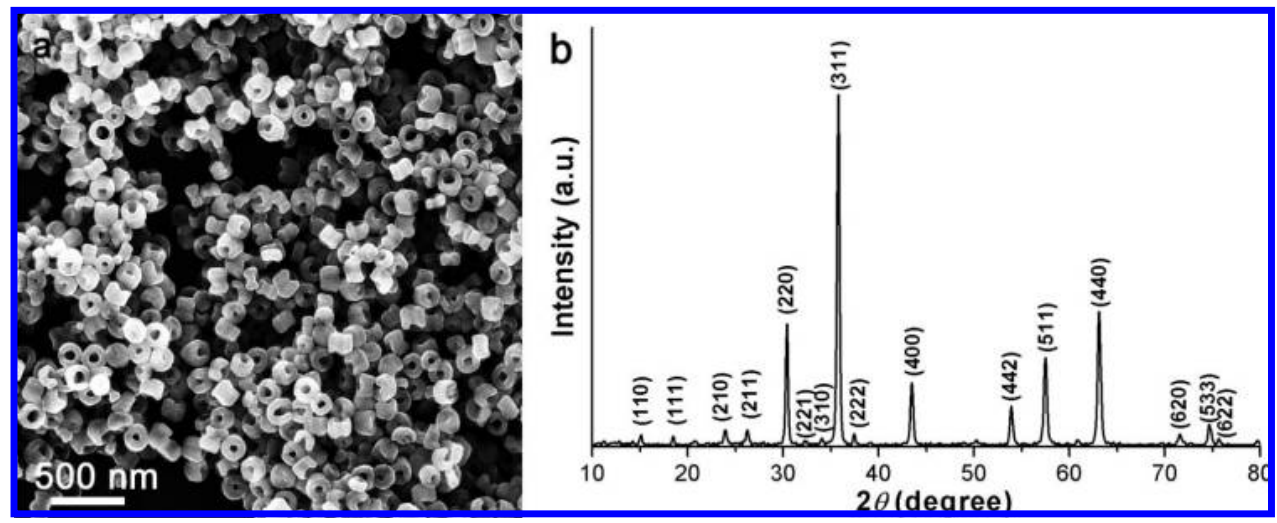

Figure 6. (a) SEM image and (b) XRD pattern of the $\gamma-\mathrm{Fe}_{2} \mathrm{O}_{3}$ nanorings. The morphology of the initial nanorings was maintained during the phase transformation from $\mathrm{Fe}_{3} \mathrm{O}_{4}$ to $\gamma-\mathrm{Fe}_{2} \mathrm{O}_{3}$.

three-dimensional magnetic configuration, which is indicative of the transition to a tubelike morphology.

2.1. Microstructural Characterization. Figure 5a,b shows SEM and TEM images of the $\mathrm{Fe}_{3} \mathrm{O}_{4}$ nanorings, where the ringshaped morphology of these products is perfectly preserved. The XRD patterns of the $\mathrm{Fe}_{3} \mathrm{O}_{4}$ (Figure 5c) confirm the high crystallinity of the products, all of which are well-indexed to the pure inverse spinel structure of magnetite (JPCDS no. 110614). The SEM images of the $\gamma-\mathrm{Fe}_{2} \mathrm{O}_{3}$ nanorings shown in Figure 6 also indicate that the nanorings can be successfully transformed. The readily indexed XRD patterns clarify the formation of inverse-spinel-structured $\gamma-\mathrm{Fe}_{2} \mathrm{O}_{3}$. In addition to the preservation of the morphology, there is almost no change in the size on conversion from $\alpha-\mathrm{Fe}_{2} \mathrm{O}_{3}$ to $\mathrm{Fe}_{3} \mathrm{O}_{4}$ and $\mathrm{Fe}_{3} \mathrm{O}_{4}$ to $\gamma-\mathrm{Fe}_{2} \mathrm{O}_{3}$, as obtained from the size distribution histograms (Figure S11 in the Supporting Information).

The apparent difference between the XRD patterns of the $\alpha-\mathrm{Fe}_{2} \mathrm{O}_{3}$ nanorings (Figure $\mathrm{S} 2 \mathrm{a}$ in Supporting Information) and $\mathrm{Fe}_{3} \mathrm{O}_{4}$ nanorings (Figure 5c) clearly shows the structural conversion from corundum to spinel. Because $\mathrm{Fe}_{3} \mathrm{O}_{4}$ has a crystal structure similar to that of $\gamma-\mathrm{Fe}_{2} \mathrm{O}_{3}$, which makes it hard to distinguish between the two on the basis of XRD patterns alone, we also used XPS, which is sensitive to the iron valence state, to analyze the two different phases. ${ }^{26}$ One important difference between $\mathrm{Fe}_{2} \mathrm{O}_{3}\left(\alpha\right.$ or $\gamma$ ) and $\mathrm{Fe}_{3} \mathrm{O}_{4}$ is that the former has satellites in the Fe 2 p core-level spectrum while the latter does not. To further prove the structural conversion from $\alpha-\mathrm{Fe}_{2} \mathrm{O}_{3}$ to $\mathrm{Fe}_{3} \mathrm{O}_{4}$, the XPS spectra for $\alpha-\mathrm{Fe}_{2} \mathrm{O}_{3}$ nanorings are also shown here. Figure 7 shows the core-level XPS patterns of the $\alpha-\mathrm{Fe}_{2} \mathrm{O}_{3}, \mathrm{Fe}_{3} \mathrm{O}_{4}$, and $\gamma-\mathrm{Fe}_{2} \mathrm{O}_{3}$ nanorings in the $\mathrm{O} 1 \mathrm{~s}$ and $\mathrm{Fe} 2 \mathrm{p}$ regions. The XPS O 1s spectra of the samples, shown in Figure 7a, all have a nearly identical single-line profile. The Fe $2 \mathrm{p}$ spectral line shapes for the $\alpha-\mathrm{Fe}_{2} \mathrm{O}_{3}$ and $\gamma-\mathrm{Fe}_{2} \mathrm{O}_{3}$ nanorings are equivalent but differ significantly from that of $\mathrm{Fe}_{3} \mathrm{O}_{4}$ nanorings. The two major components at binding energies of 711.2 and $724.5 \mathrm{eV}$ accompanied by a satellite are attributed to $2 \mathrm{p}_{3 / 2}$ and $2 \mathrm{p}_{1 / 2}$ core levels for $\alpha-\mathrm{Fe}_{2} \mathrm{O}_{3}$ and $\gamma-\mathrm{Fe}_{2} \mathrm{O}_{3}$ nanorings. In the XPS pattern of the $\mathrm{Fe}_{3} \mathrm{O}_{4}$ nanorings, the broad $\mathrm{Fe} 2 \mathrm{p}$ signals are attributed to the coexistence of $\mathrm{Fe}^{3+}$ and $\mathrm{Fe}^{2+}$ states, and in addition, no satellites can be identified, excluding the presence of $\gamma-\mathrm{Fe}_{2} \mathrm{O}_{3}$ or $\alpha-\mathrm{Fe}_{2} \mathrm{O}_{3}$ in the $\mathrm{Fe}_{3} \mathrm{O}_{4}$ samples.

Figure 8 shows TEM images, SAED patterns, and HRTEM images of $\mathrm{Fe}_{3} \mathrm{O}_{4}$ nanorings. The SAED patterns indicate that

(26) Fujii, T.; de Groot, F. M. F.; Sawatzky, G. A.; Voogt, F. C.; Hibma, T.; Okada, K. Phys. Rev. B 1999, 59, 3195-3202. 


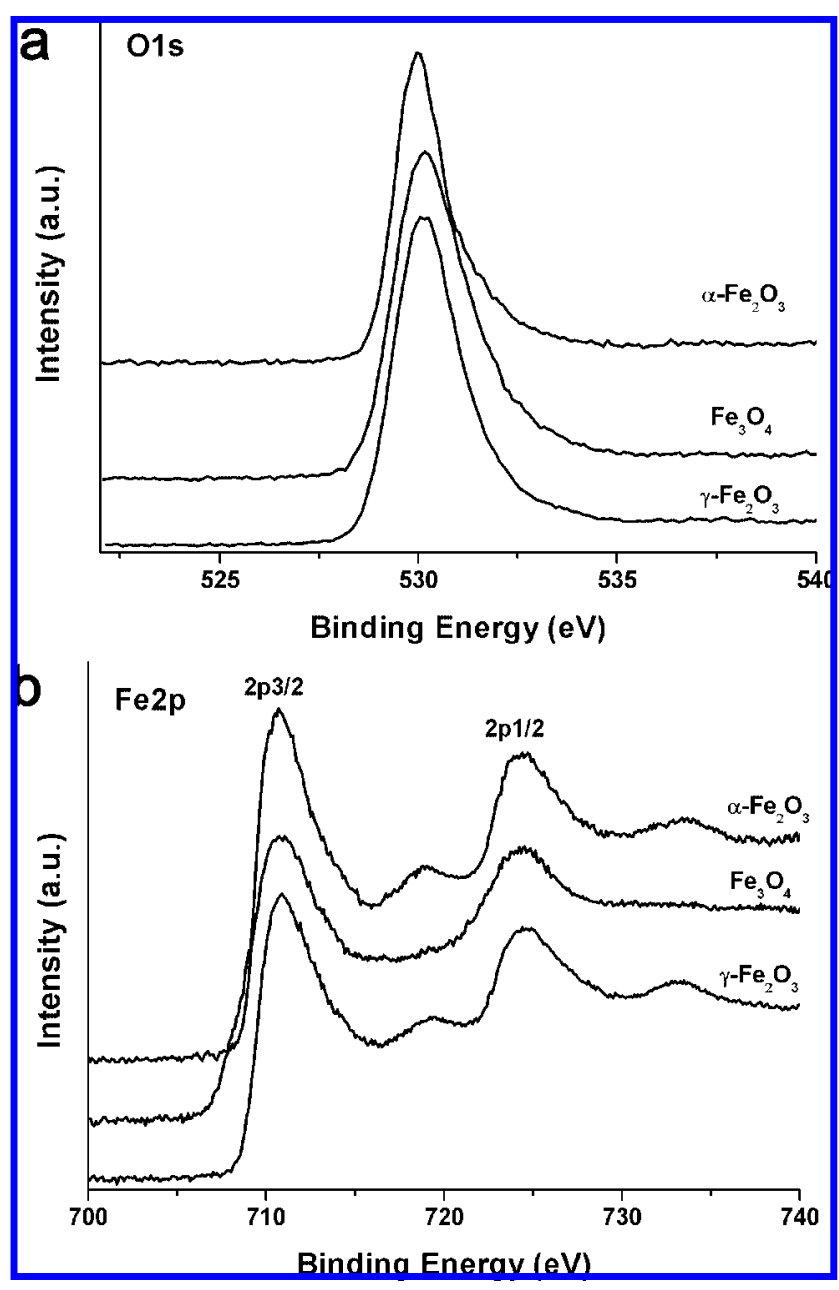

Figure 7. (a) $\mathrm{O} 1 \mathrm{~s}$ and (b) $\mathrm{Fe} 2 \mathrm{p}$ core-level spectra of the $\alpha-\mathrm{Fe}_{2} \mathrm{O}_{3}, \mathrm{Fe}_{3} \mathrm{O}_{4}$, and $\gamma-\mathrm{Fe}_{2} \mathrm{O}_{3}$ nanorings.

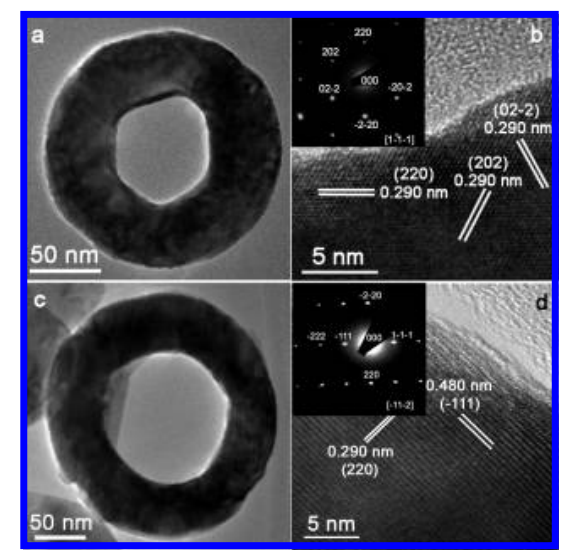

Figure 8. (a, c) TEM images and (b, d) HRTEM images and (insets) SAED patterns of $\mathrm{Fe}_{3} \mathrm{O}_{4}$ nanorings, showing two growth orientations: $(\mathrm{a}, \mathrm{b})$ ring axis of $<111>$; (c, d) ring axis of $<112>$.

the $\mathrm{Fe}_{3} \mathrm{O}_{4}$ nanorings are single-crystalline, a property inherited from the $\alpha-\mathrm{Fe}_{2} \mathrm{O}_{3}$ nanorings, while two crystallographic orientations, $<111>$ and $<112>$, are observed for the $\mathrm{Fe}_{3} \mathrm{O}_{4}$ nanorings, as shown in Figure 8. Therefore, during the phase transformation from $\alpha-\mathrm{Fe}_{2} \mathrm{O}_{3}$ to $\mathrm{Fe}_{3} \mathrm{O}_{4}$, although the single-crystal nature is well-preserved, two orientation conversion relationships exist: $[001]_{\alpha-\mathrm{Fe}_{2} \mathrm{O}_{3}} \rightarrow[111]_{\mathrm{Fe}_{3} \mathrm{O}_{4}}$ and $[001]_{\alpha-\mathrm{Fe}_{2} \mathrm{O}_{3}} \rightarrow[112]_{\mathrm{Fe}_{3} \mathrm{O}_{4}}$. The former one is in accordance with the previous results, ${ }^{27}$ indicating that such a phase transformation is a topotactic one. ${ }^{28}$ The structural transformation of $[001]_{\alpha-\mathrm{Fe}_{2} \mathrm{O}_{3}}$ to $[112]_{\mathrm{Fe}_{3} \mathrm{O}_{4}}$ has never been observed directly by electron microscopy, though similar results based on X-ray diffraction measurements have been reported. ${ }^{29}$ To characterize the local environment and distribution of $\mathrm{Fe}$ atoms in the $\mathrm{Fe}_{3} \mathrm{O}_{4}$ nanorings, high-resolution Fe $L_{2,3}$-edge absorption spectroscopy of different nanorings using STXM was performed. Figure 9a shows the Fe element intensity map obtained from the $\mathrm{Fe} L_{3}$-edge transmission image of a nanoring sample. The differences in intensity of the Fe mapping can be attributed to the varying thickness of the sample. NEXAFS spectra extracted from three different $\mathrm{Fe}_{3} \mathrm{O}_{4}$ nanorings are compared in Figure 9b. All of the spectra show the characteristic absorption peaks of magnetite $\left(\mathrm{Fe}_{2} \mathrm{O}_{3} \cdot \mathrm{FeO}\right)$ resulting from $\mathrm{Fe}^{2+}$ on octahedral sites and $\mathrm{Fe}^{3+}$ on both octahedral and tetrahedral sites. ${ }^{30}$ The lack of an energy shift in the spectra suggests that the $\mathrm{Fe}$ atoms are homogeneously distributed without change of valence.

2.2. Magnetic Properties of the $\mathrm{Fe}_{3} \mathrm{O}_{4}$ Nanorings. Figure 10a displays the field dependence of the magnetization of the $\mathrm{Fe}_{3} \mathrm{O}_{4}$ nanorings. The $\mathrm{Fe}_{3} \mathrm{O}_{4}$ nanorings are ferrimagnetic at room temperature, with a coercivity of 120 Oe. The saturation magnetization $\left(M_{\mathrm{S}}\right)$ of the magnetite rings is $87.4 \mathrm{emu} / \mathrm{g}$, which is just a little smaller than the corresponding bulk value (92 $\mathrm{emu} / \mathrm{g}^{31}$ for $\mathrm{Fe}_{3} \mathrm{O}_{4}$ ), which may be attributed to the increase in surface effects with decreasing particle size. ${ }^{32}$ There are two kinks near zero magnetization in the $M-H$ loop, which indicate the possibility of two vortex states with opposite directions, as shown schematically in the insets of Figure $10 \mathrm{a} .{ }^{33}$ The temperature dependence of magnetization of the $\mathrm{Fe}_{3} \mathrm{O}_{4}$ nanorings was measured by applying a magnetic field of 50 Oe and measuring zero-field-cooled (ZFC) and field-cooled (FC) curves (Figure 10b) to determine the characteristic Verwey transition temperature $\left(T_{\mathrm{V}}\right)$. For bulk $\mathrm{Fe}_{3} \mathrm{O}_{4}, T_{\mathrm{V}} \approx 119 \mathrm{~K}$, above which fast electron hopping between the $\mathrm{Fe}^{2+}$ and $\mathrm{Fe}^{3+}$ ions on the octahedral $B$ sites occurs. ${ }^{34}$ The ZFC curve of the $\mathrm{Fe}_{3} \mathrm{O}_{4}$ nanorings shows two transition temperatures: one is at $\sim 100$ $\mathrm{K}$, corresponding to the Verwey transition, and the other is located at $\sim 50 \mathrm{~K}$, implying the occurrence of spin reorientation. ${ }^{35}$ It should be noted that the FC behavior is different from that of bulk $\mathrm{Fe}_{3} \mathrm{O}_{4}$. This is consistent with other FC measurements on $\mathrm{Fe}_{3} \mathrm{O}_{4}$ nanostructures. ${ }^{36,37}$

Figure 11a shows an off-axis electron hologram ${ }^{38}$ of a single $\mathrm{Fe}_{3} \mathrm{O}_{4}$ nanoring with a length of $50 \mathrm{~nm}$ along the ring axis, an

(27) (a) Varanda, L. C.; Jafelicci, M., Jr.; Tartaj, P.; O’Grady, K.; GonzálezCarreño, T.; Morales, M. P.; Muñoz, T.; Serna, C. J. J. Appl. Phvs. 2002, 92, 2079-2085. (b) Morales, M. P.; Pecharroman, C.; GonzálezCarreño, T.; Serna, C. J. J. Solid State Chem. 1994, 108, 158-163.

(28) Feitknecht, W.; Mannweiler, U. Helv. Chim. Acta 1967, 50, 570581.

(29) Bursill, L. A.; Withers, R. L. J. Appl. Crystallogr. 1979, 12, 279286.

(30) Huang, D. J.; Chang, C. F.; Jeng, H. T.; Guo, G. Y.; Lin, H. J.; Wu, W. B.; Ku, H. C.; Fujimori, A.; Takahashi, Y.; Chen, C. T. Phvs. Rev. Lett. 2004, 93, 077204-1-077204-4.

(31) Smit, J.; Wijn, H. P. Ferrie; Wiley: New York, 1959; p 369.

(32) Valstyn, E. P.; Hanton, J. P.; Morrish, A. H. Phvs. Rev. 1962, 128, 2078-2087.

(33) Zhu, F. Q.; Fan, D. L.; Zhu, X. C.; Zhu, J. G.; Cammarata, R. C.; Chien, C. L. Adv. Mater. 2004, 16, 2155-2159.

(34) Verwey, E. J. W. Nature 1939, 144, 327-328.

(35) Yang, J. B.; Zhou, X. D.; Yelon, W. B.; James, W. J.; Cai, Q.; Gopalakrishnan, K. V.; Malik, S. K.; Sun, X. C.; Nikles, D. E. J. Appl. Phvs. 2004, 95, 7540-7542.

(36) Tang, J. K.; Wang, K. Y.; Zhou, W. L. J. Appl. Phvs. 2001, 89, 76907692 . 


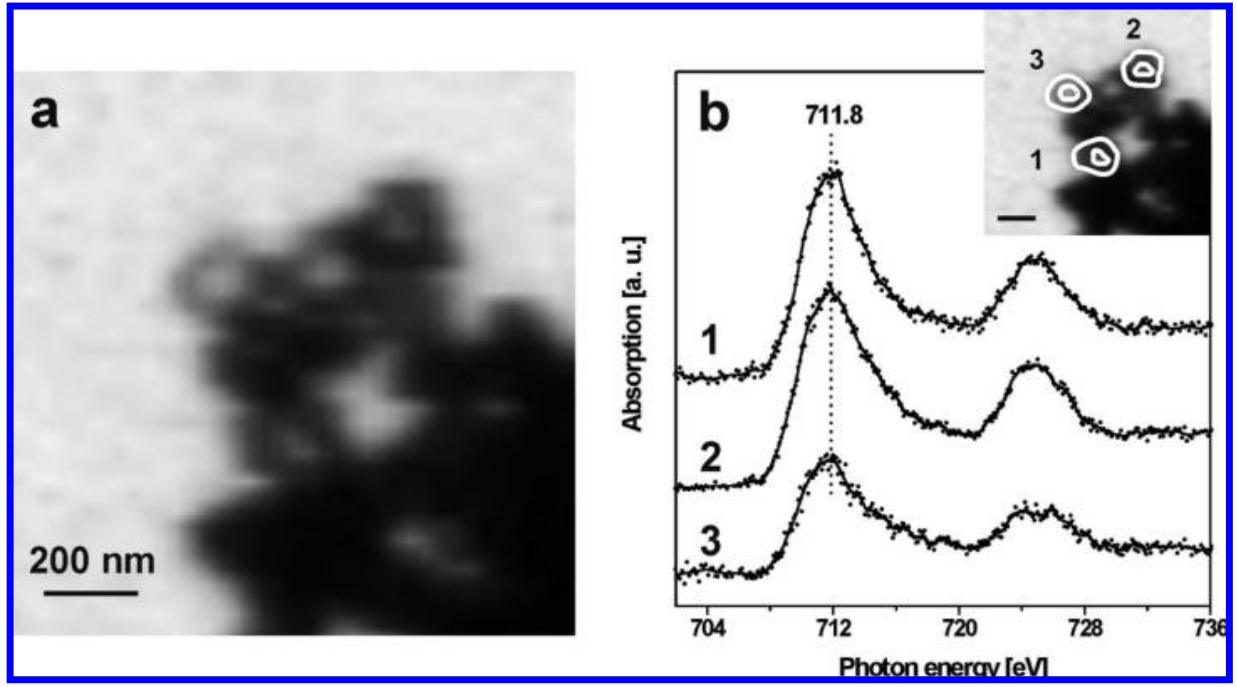

Figure 9. (a) STXM transmission image (Fe element mapping) of $\mathrm{Fe}_{3} \mathrm{O}_{4}$ nanorings obtained at the $\mathrm{Fe} L_{3}$-edge absorption (711.8 eV). (b) Comparison of the $\mathrm{Fe} L_{2,3}$-edge NEXAFS spectra extracted from the three different nanorings indicated in the inset.

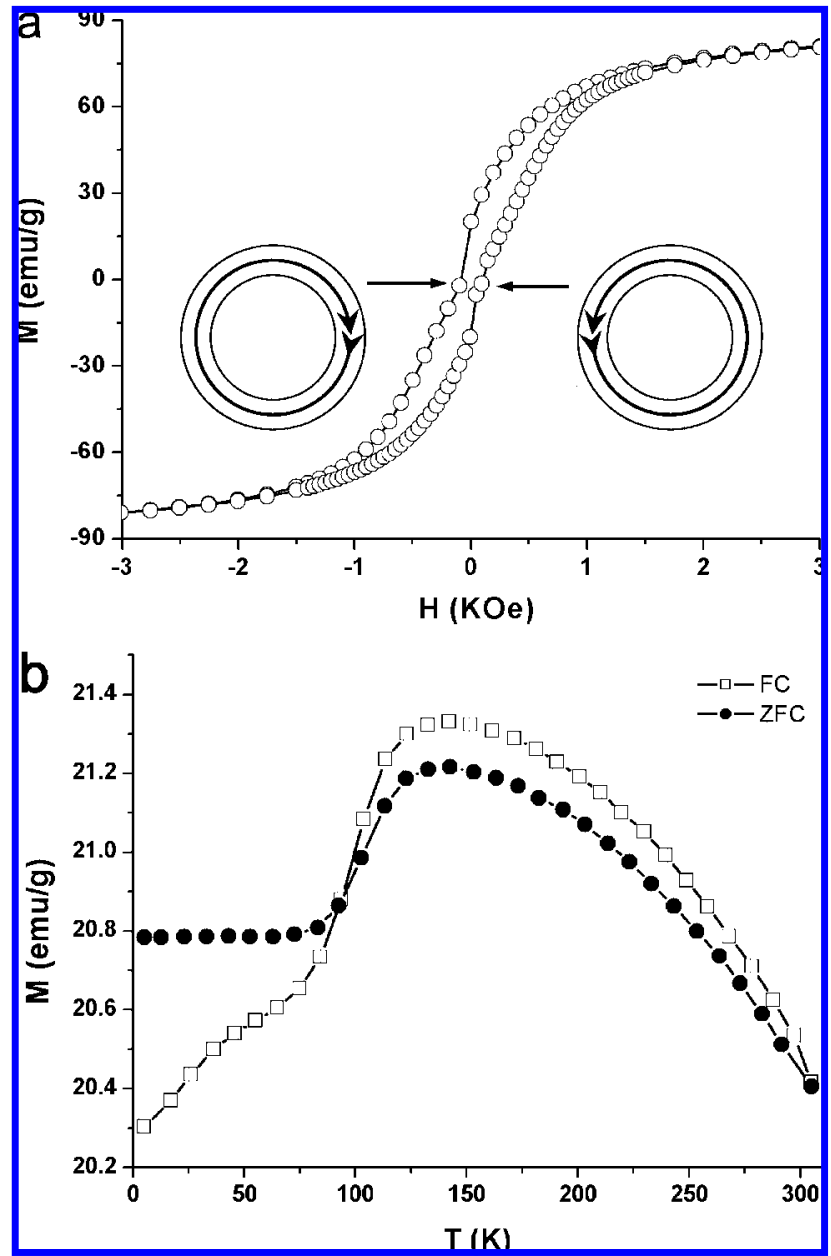

Figure 10. (a) Hysteresis loop of the $\mathrm{Fe}_{3} \mathrm{O}_{4}$ nanorings at $300 \mathrm{~K}$, and (b) zero-field-cooled (ZFC) and field-cooled (FC) curves of $\mathrm{Fe}_{3} \mathrm{O}_{4}$ nanorings. Insets of (a): schematic illustrations of the vortex state in the magnetite ring at remanence.

outer diameter of $160 \mathrm{~nm}$, and an averaged inner diameter of $85 \mathrm{~nm}$. Figure 11b displays the corresponding magnetic induction map, shown in the form of contours. The ring has a vortex state with minimal external stray fields, where magnetic

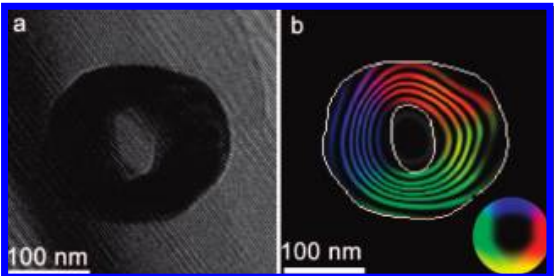

Figure 11. (a) Off-axis electron hologram of a single magnetite ring with an average thickness of $50 \mathrm{~nm}$. In the form of bending interference fringes, the phase changes can be seen. (b) Direction of the magnetic induction under field-free conditions following magnetization, indicated by color as shown in the color wheel in the inset (red $=$ right, yellow $=$ down, green $=$ left, blue $=$ up).

moments circulate around the ring. From the HRTEM analysis, we found that the $\mathrm{Fe}_{3} \mathrm{O}_{4}$ nanorings have two different crystallographic orientations, $<111>$ and $<112>$, both of which show similar magnetic vortex states. This indicates that it is the ring geometry rather than the magnetocrystalline anisotropy that controls the magnetic configuration of the $\mathrm{Fe}_{3} \mathrm{O}_{4}$ nanorings.

When the ring thickness is increased by controlling the synthesis conditions, this two-dimensional $\mathrm{Fe}_{3} \mathrm{O}_{4}$ ring structure becomes more three-dimensional (tubelike) as the ring thickness becomes comparable with ring diameter. In a thick ring with a strong variation in thickness, we have observed a state that is intermediate between the in-plane vortex and the out-of-plane tube state. ${ }^{25}$ In the thinner parts of the ring, the magnetization points in-plane, while in the thicker parts of the ring, a magnetic state with several vortices inside the ring itself and several magnetic domains are visible. ${ }^{25}$ A comparison of the threedimensional reconstructed electron tomography and the magnetic induction map indicates that the vortices are at the regions of the largest thickness, where the magnetic spins can point along the tube length.

\section{Conclusions}

We have presented a novel approach for synthesizing singlecrystal $\alpha-\mathrm{Fe}_{2} \mathrm{O}_{3}$ nanorings, employing a double anion-assisted hydrothermal method. Here the cooperative action of the phosphate and sulfate ions, involving adsorption and coordination, is a crucial factor in bringing about the formation of the nanorings. By varying the ratios of phosphate and sulfate ions 
to ferric ions, we have been able to produce a series of hollow nanostructures with tunable size, morphology, and surface architecture. These hollow $\alpha-\mathrm{Fe}_{2} \mathrm{O}_{3}$ nanostructures can then be converted via a reduction or reduction-oxidation process to single-crystal $\mathrm{Fe}_{3} \mathrm{O}_{4}$ or $\gamma-\mathrm{Fe}_{2} \mathrm{O}_{3}$, respectively, having exactly the same morphology. While the $\mathrm{Fe}_{3} \mathrm{O}_{4}$ nanorings have two different crystallographic directions, both display a similar magnetic flux closure vortex state, indicating that it is the geometry rather than the crystal orientation that controls the magnetic configuration. This solution-based route provides an important large-scale method of preparing high-quality, tailormade iron oxide nanostructures. This source of highly functional three-dimensional nanostructures will trigger their use in a wide

(37) Chang, M. T.; Chou, L. J.; Hsieh, C. H.; Chueh, Y. L.; Wang, Z. L.; Yasukazu, M.; Daisuke, S. Adv. Mater. 2007, 19, 2290-2294.

(38) Kläui, M.; Vaz, C. A. F.; Lopez-Diaz, L.; Bland, J. A. C. J. Phys.: Condens. Matter 2003, 15, R985-R1024. range of applications, including carriers for biomagnetic sensing, medical diagnostics and treatment, magnetic data storage, and catalysis.

Acknowledgment. This work was supported by NSFC (20671005, 20423005, and 20221101), NSFC\&RGC (20610068), the MOST of China (2006CB601104), the Founder Foundation of PKU, and the Deutsche Forschungsgemeinschaft. M.E. acknowledges support by the DAAD, and R.E.D.-B. acknowledges support by the Royal Society. Part of this work was carried out at the Swiss Light Source, Paul Scherrer Institut, Villigen, Switzerland.

Supporting Information Available: XRD patterns of the $\alpha-\mathrm{Fe}_{2} \mathrm{O}_{3}$ nanorings, additional SEM images, HRTEM images, schematic illustrations of the crystal structure of $\alpha-\mathrm{Fe}_{2} \mathrm{O}_{3}$, and particle size distribution histograms for $\alpha-\mathrm{Fe}_{2} \mathrm{O}_{3}, \mathrm{Fe}_{3} \mathrm{O}_{4}$, and $\gamma-\mathrm{Fe}_{2} \mathrm{O}_{3}$ nanorings. This material is available free of charge via the Internet at http://pubs.acs.org.

JA805152T 